\title{
Intravitreal Bevacizumab for the Treatment of Chronic or Recurrent Central Serous Chorioretinopathy
}

\author{
Mitzy E. Torres Soriano1,2, Gerardo García Aguirre3, Maximiliano Gordon Angelozzi, \\ Veronica Kon Jara ${ }^{5}$, Jans Fromow Guerra ${ }^{3}$, Michael D. Ober ${ }^{6}$, Hugo Quiroz Mercado ${ }^{7}$ \\ ${ }^{1}$ Unidad Oftalmológica “Dr Torres López”, Centro Médico Cagua, Cagua, Venezuela \\ ${ }^{2}$ Instituto de Especialidades Oftalmológicas del Centro (IDEOF), Maracay, Venezuela \\ ${ }^{3}$ Retina Department, Asociación para evitar la Ceguera, Hospital Dr. Luis Sanchez Bulnes, Mexico City, Mexico \\ ${ }^{4}$ Centro de la Visión Gordon Manavella, Rosario, Argentina \\ ${ }^{5}$ Department of Ophthalmology, University of North Carolina, Chapel Hill, USA \\ ${ }^{6}$ Retina Consultants of Michigan, Southfield, USA \\ ${ }^{7}$ Ophthalmology Service at Denver Health Medical Center, Denver, USA \\ Email: mitzytorres@yahoo.com
}

Received 16 May 2014; revised 29 June 2014; accepted 16 July 2014

Copyright (C) 2014 by authors and Scientific Research Publishing Inc.

This work is licensed under the Creative Commons Attribution International License (CC BY). http://creativecommons.org/licenses/by/4.0/

(c) (i) Open Access

\begin{abstract}
Background: We report the use of intravitreal bevacizumab as an option for the treatment of chronic or recurrent central serous chorioretinopathy (CSC). Methods: Eight eyes with chronic or recurrent CSC received intravitreal bevacizumab $(1.25 \mathrm{mg} / 0.05 \mathrm{cc})$ and underwent best corrected visual acuity (VA), optical coherent tomography (OCT), fluorescein angiography (FA) and indocyanine green angiography (ICG) before, and one, three and six months after treatment. Results: All patients showed improvement in visual acuity, fluorescein angiographic leakage, choroidal hyperpermeability and reduced or resolved neurosensory detachment following treatment. Two patients require a second dose of intravitreal bevacizumab. Conclusions: Intravitreal injection of bevacizumab was associated with visual improvement and reduced neurosensory detachment without adverse events in patients with chronic or recurrent CSC. Although these results are promising, further investigations would be helpful to understand this therapy for patients with CSC.
\end{abstract}

\section{Keywords}

Central Serous Chorioretinopathy, Intravitreal Bevacizumab, Retinal Pigment Epithelium Detachment

How to cite this paper: Torres Soriano, M.E., García Aguirre, G., Gordon Angelozzi, M., Kon Jara, V., Fromow Guerra, J., Ober, M.D. and Quiroz Mercado, H. (2014) Intravitreal Bevacizumab for the Treatment of Chronic or Recurrent Central Serous Chorioretinopathy. Open Journal of Ophthalmology, 4, 57-64. http://dx.doi.org/10.4236/ojoph.2014.43010 


\section{Introduction}

Central serous chorioretinopathy (CSC) is a well-characterized disorder leading to serous neurosensory elevation of the central macula. The acute form of the disease is associated with focal leakage at the level of the retinal pigment epithelium (RPE) demonstrated with fluorescein angiography (FA). Fortunately, the disorder is self-limited in the majority of patients, who also regain excellent vision. Occasionally, the neurosensory detachment persists and leads to pigment epithelial and photoreceptor damage with visual impairment.

Chronic cases of CSC may yield further complications such as diffuse retinal pigment epithelial descompensation, subretinal precipitates, descending atrophic tracts, dependent retinal detachment, cystoid macular edema, cystoid macular degeneration, secondary choroidal neovascularization, and fibrous scarring [1]. Although scatter thermal laser photocoagulation may shorten the duration of macular detachment in chronic CSC, the reported cases are few and lack long-term follow-up [2].

The increasing use of indocyanine green angiography (ICG) in CSC has improved the understanding of the pathogenesis of CSC and has demonstrated that CSC primarily affects the choroidal circulation, resulting in multifocal areas of choroidal vascular hyperpermeability [3]-[5]. Recently, researchers have reported favorable results when treating chronic CSC with indocyanine green guided photodynamic therapy (PDT) using verteporfin. Verteporfin produces alteration of choriocapillary blood flow that may reverse or prevent fluid leakage beneath and beyond the RPE in patients with chronic CSC [1]. However, the application of conventional PDT in CSC is not without its complications as the development of RPE atrophy, choriocapillary ischemia, and secondary choroidal neovascularization (CNV) have also been reported after PDT for chronic CSC [6]-[8].

The purpose of this article is to report the use of intravitreal bevacizumab as a valuable option of treatment for patients with chronic or recurrent CSC.

\section{Materials and Methods}

This study was a prospective, nonrandomized, consecutive, open-labeled interventional case series conducted in the Retina Service of "Dr. Luis Sánchez Bulnes” Hospital from the "Asociación Para Evitar la Ceguera en México”, from March 2006 to February 2008.

Patients were evaluated for the presence of chronic or recurrent CSC with visual disturbance, defined as idiopathic elevation of the neurosensory retina, associated with pigment epithelial detachment (PED) who complained of decreased visual acuity for at least six months or had more than one episode in the presence of focal leaks at the level of the RPE on FA.

Patients with any range of visual acuity, presence of subretinal fluid (SRF) and/or serous PED involving the fovea on optical coherence tomography (OCT) were included, who had presence of active angiographic leakage in FA caused by CSC but not CNV or other disease and abnormal dilated choroidal vasculature and other features in ICG consistent with the diagnosis of CSC. Patients who received previous PDT or focal thermal laser photocoagulation for the treatment of CSC or had evidence of CNV, polypoidal choroidal vasculopathy, or other maculopathy on clinical examination, FA, or ICG were excluded. Informed consent was obtained from all subjects. After informed consent and discussion of therapeutic options, patients were treated with intravitreal injection of $0.05 \mathrm{ml}$ (1.25 mg) of bevacizumab, $4 \mathrm{~mm}$ posterior to the limbus, in the superotemporal quadrant under aseptic conditions.

Each patient underwent best-corrected visual acuity (BCVA) measurements (Snellen or Early Treatment Diabetic Retinopathy Study charts), a dilated fundus examination, optical coherence tomography, fluorescein angiography and indocyanine green angiography at baseline, one, three and six months after injection of intravitreal bevacizumab.

Data obtained were analyzed with frequency and descriptive statistics. The Wilcoxon signed ranks test was performed to assess change in BCVA from baseline to the interval examination 6 months after treatment. TheSPSS software (version 12.0, SPSS, Inc., Chicago, IL) was used for data analysis.

\section{Results}

Table 1 summarizes the clinical characteristics of the patients and treatment results. Eight eyes of 8 patients (5 men, 3 women) with chronic or recurrent CSC were treated with intravitreal bevacizumab $(1.25 \mathrm{mg} / 0.05 \mathrm{ml})$. The age at the time of treatment ranged from 28 to 53 years (mean 39). Pretreatment BCVA ranged from counting fingers at one meter to 20/30 (median 20/100). All patients had decreased vision for at least six months or recent exacerbation of visual changes before receiving treatment and had one or more focal RPE leaks re- 
Table 1. Summary of the clinical findings.

\begin{tabular}{|c|c|c|c|c|c|c|c|}
\hline Patient No & Age/Sex & $\begin{array}{l}\text { Number of } \\
\text { Injections }\end{array}$ & $\begin{array}{l}\text { VA pre } \\
\text { Avastin }\end{array}$ & $\begin{array}{l}\text { Final VA (post } \\
\text { Avastin) }\end{array}$ & $\begin{array}{l}\text { Foveal Thickness } \\
(\mu \mathrm{m}) \text { Pre Avastin }\end{array}$ & $\begin{array}{l}\text { Foveal Thickness } \\
(\mu \mathrm{m}) \text { Post Avastin }\end{array}$ & $\begin{array}{l}\text { Follow up } \\
\text { (months) }\end{array}$ \\
\hline 1 & 29:M & 2 & $20 / 1250$ & $20 / 60$ & 254 & 188 & 6 \\
\hline 2 & 29:F & 1 & $20 / 30$ & $20 / 20$ & 428 & 210 & 6 \\
\hline 3 & 49:M & 1 & $20 / 60$ & $20 / 40$ & 288 & 212 & 6 \\
\hline 4 & 44:M & 1 & $20 / 30$ & $20 / 20$ & 394 & 170 & 6 \\
\hline 5 & 41:F & 2 & $20 / 1250$ & $20 / 200$ & 385 & 317 & 3 \\
\hline 6 & 53:M & 1 & $20 / 50$ & $20 / 50$ & 383 & 333 & 1 \\
\hline 7 & 28:F & 1 & $20 / 30$ & $20 / 25$ & 278 & 202 & 1 \\
\hline 8 & 37:M & 1 & $20 / 40$ & $20 / 30$ & 506 & 296 & 3 \\
\hline
\end{tabular}

sponsible for neurosensory detachment, identified as pinpoint areas of hyperfluorescence on early FA frames that increased in size through later frames. Some patients had localized fine mottling and clumping of the pigment in the retina pigment epithelium.

Visual acuity improved from $20 / 100$ at baseline to $20 / 70$ at one month $(p=0.023$ ), to 20/50 at three months ( $p$ $=0.026)$, to $20 / 30$ at six months $(p=0.059)$. Optic coherence tomography showed improvement in the macular anatomy, resolution (Figure 1) or decrease of subretinal fluid, with a change in central retinal thickness from $364.50 \mu$ before treatment to $257 \mu$ at one month $(p=0.012), 221.83 \mu$ at three months $(p=0.028)$ and $195 \mu$ at six months $(p=0.068)$. These differences were statistically significant $(p<0.05)$.

Fluorescein angiography showed decrease or resolution of leakage areas. Indocyanine green angiography demonstrated improved midframe hyperfluorescence corresponding to areas where focal RPE leaks were seen on FA (Figure 2 and Figure 3). Two patients were given a second intravitreal injection of bevacizumab. After treatment, no serious ocular or systemic effects were observed.

\section{Discussion}

Finding an optimal treatment for CSC is a complicated matter, since spontaneous resolution occurs in most patients, and emotional factors play an important role in the pathogenesis of the disease. Several treatments have been reported with good results, but interpretation of the results becomes difficult since it is hard to distinguish if the good result is due to the natural course of the disease, the placebo effect, or an actual response to treatment. When dealing with chronic CSC, the role of spontaneous resolution or the placebo effect may be less but can still be significant.

Various medical treatments have been attempted for this disorder, including acetazolamide, $\beta$-blockers, vitamins, and non-steroidal anti-inflamatory medications without decisive benefit [9]. Laser photocoagulation has also been reported to accelerate resolution of the neurosensory detachment in CSC when it is applied on focal sites of RPE leakage [10] [11]. The efficacy, however, is questionable when ill-defined areas of RPE decompensation are present, as in chronic disease. Regression of subretinal exudation was obtained by laser photocoagulation in selected cases of chronic CSC when the treatment was performed in a grid or confluent pattern such as to produce closure of the choriocapillaris in the areas with decompensated RPE [12]-[14].

Several case series have reported the use of indocyanine green guided photodynamic therapy to treat chronic CSC, defined as $>6$ months duration of disease with diffuse decompensation of the RPE, but lacking focal FA leaks [15] [16]. 


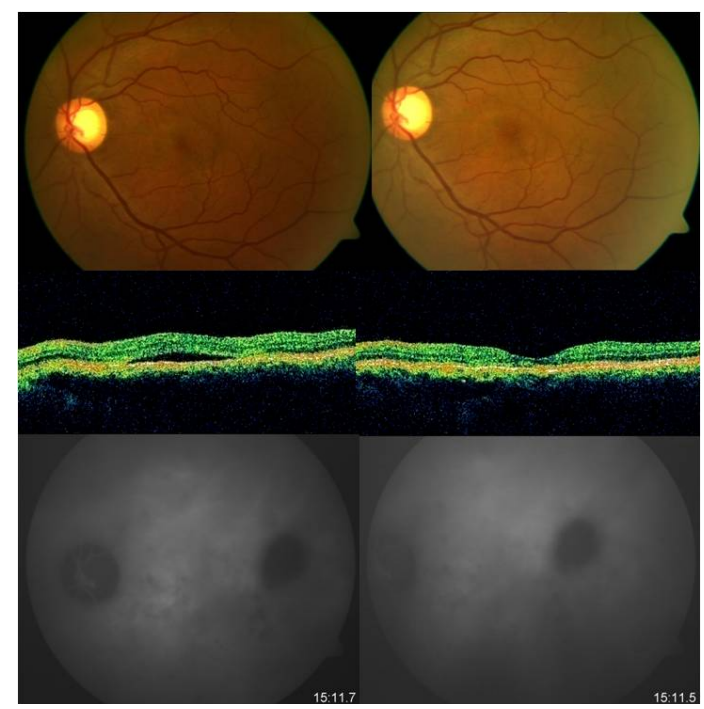

Figure 1. Patient with recurrent CSC in the left eye. Pretreatment (left side) OCT confirmed the presence of a serous neurosensory detachment under the fovea. ICG angiography revealed a plaque of hyperfluorescence in the center of the macula, and a hypofluorescent area in the temporal macula. After treatment (right side) there is resolution of fluid in OCT and ICG angiography hyperfluorescence, although the hypofluorescent area in the temporal macula remains.

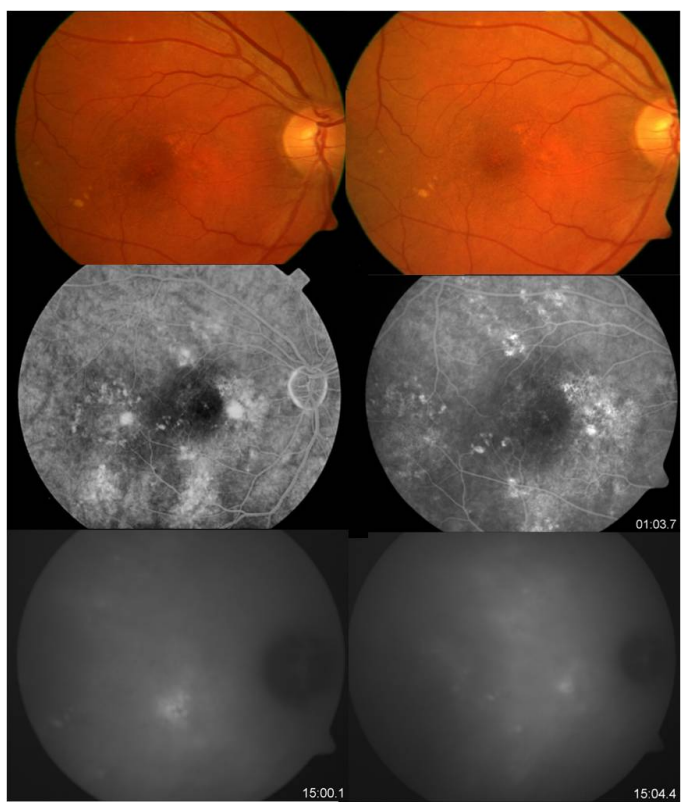

Figure 2. Pre-treatment (left side) Fundus image of chronic central serous chorioretinopathy. FA revealed multiple perifoveal spots of hyperfluorescence, and a mottled hyper-hypofluorescent pattern caused by atrophic changes of RPE. ICG angiography shows various plaques of hyperfluorescence (choroidal hyperpermeability). After treatment (right side) the hyperfluorescence in FA and ICG angiography improved significantly. 


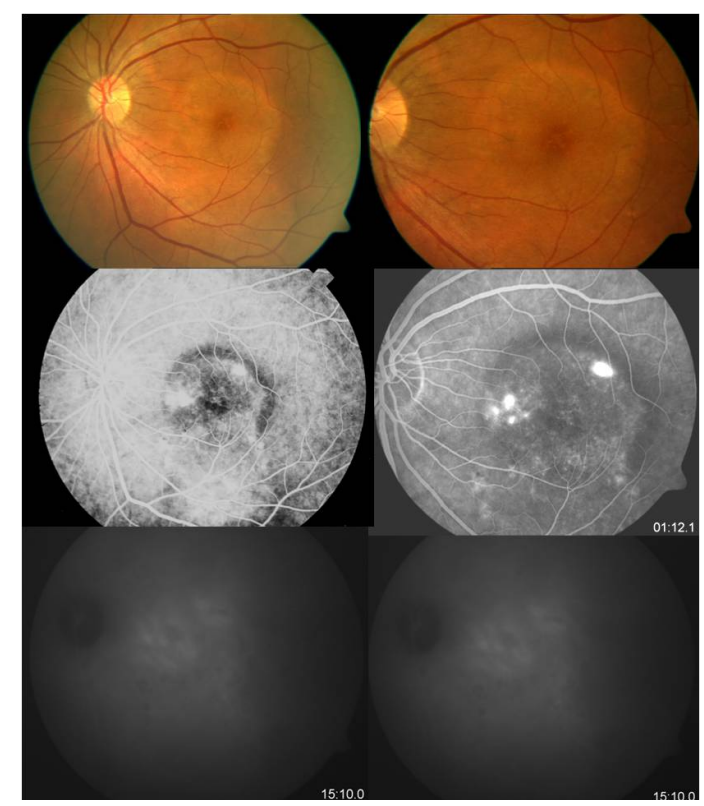

Figure 3. Chronic CSC unresponsive to intravitreal bevacizumab. FA showed multiple pinpoint areas of hyperfluorescence and ICG angiography confirmed abnormal fluorescence in the same area. There is no evident change before (left side) and after treatment (right side) with intravitreal bevacizumab.

Cardillo Piccolino et al. performed ICG-guided PDT in 16 eyes with chronic CSC and treatment resulted in complete resolution of serous retinal detachment one month after treatment in $75 \%$ of eyes. At three months after PDT, $69 \%$ of eyes had visual improvement of 1 or more lines. In this study $31 \%$ of eyes developed secondary RPE changes at the site of PDT which were thought to be due to hypoxic damage caused by choriocapillaris occlusion [6]. The development of CNV after PDT for chronic CSC has also been reported in previous case series and a case report by Colucciello [7] [8]. These potential side effects might be important considerations to restrict the extensive use of PDT for chronic CSC since these patients generally have better vision compared with those with exudative maculopathy secondary to CNV. Moreover, the use of various electrophysiologic tests such as multifocal electroretinography, pattern electroretinography, and electrooculography have also demonstrated objectively that PDT might result in transient impairment in retinal function following treatment [17]-[20].

In an effort to reduce collateral damage less intensive strategies have been employed with reduced fluence PDT. Chan et al. performed a randomized controlled trial of half dose PDT vs placebo and demonstrated that 95\% of treated patients versus $58 \%$ of controls had complete absence of fluid at one year visual acuity was also significantly improved [21]. However, in a recent study, Lim et al. suggested an absence of association between laser fluence settings and VA outcomes [22].

Bevacizumab is a full length antibody that binds all isoforms of VEGF developed for the treatment of sometypes of cancer. Intravitreal bevacizumab has not been tested in a randomized, controlled fashion for CSC; however, a growing number of reports in the literature support its safety and efficacy. Niegel et al. report a 57 year old female patient who received a single intravitreal injection of bevacizumab led to a rapid morphologic and functional restitution without relapse or complication during the 19 weeks period after injection and were the first to suggest that intravitreal injection of bevacizumab could be a therapeutic option for the treatment of chronic CSC [23].

Artunay and colleagues conducted a prospective, controlled, open-label, non-randomized, trial of a single 2.5 mg intravitreal bevacizumab injection in 30 previously untreated eyes with chronic CSC. Fifteen eyes of 15 patients were treated with intravitreal injections of $2.5 \mathrm{mg}(0.1 \mathrm{ml})$ bevacizumab (treatment group). Fifteen eyes of 15 patients with the same characteristics who declined treatment were an acceptable control group. Twelve 
(80\%) eyes in the treatment group compared with 8 (53.3\%) eyes in the control group showed morphologic restitution at 6 months $(p<0.01)$ [24].

In the present study, recovery or preservation of visual acuity was present in all patients, resolution or diminished of subretinal fluid by OCT and improved fluorescein leakage and choroidal hyperpermeability. We do not know the mechanism by which intravitreal bevacizumab works in CSC; however, it may be related to its ability to affect vascular permeability. The pathophysiology is also poorly understood, but the advent of indocyanine green angiography presented evidence of choroidal involvement in the disease.

Lijima H. et al. hypothesized that the choroidal circulatory disturbance in CSC is caused by impaired fibrinolysis and the resulting thrombotic occlusion in the choroidal veins. Small, localized ischemic regions caused by non-perfusion or vasoconstriction or the choriocapillaris may induce collateral choriocapillary congestion around this region. The choroidal hyperpermeability resulting from vessel congestion may be observed as localized hyperfluorescence on ICG angiography [25]. Although VEGF levels are not elevated in aqueous samples from CSC eyes, some have hypothesized that hypoxic conditions in the choroid or RPE could lead to compartmentalized VEGF expression not detected in aqueous samples. Given this hypothesis and the remarkable success of these drugs in other disorders, numerous small trials have been undertaken.

VEGF was formerly known as "vascular permeability factor" and has profound effects on vascular permeability. It follows that VEGF blockade may ameliorate the choroidal hyperpermeability present in CSC. There is some controversy regarding the ability of bevacizumab to penetrate the retina and reach the choroid, however reports suggest that it indeed does so [26], which supports the possibility that an intravitreal injection of bevacizumab may be biologically active in areas of the choroid.

There is no data available to support or refute this hypothesis. Nevertheless, the improvement in RPE and neurosensory detachments, visual acuity as well as leakage areas in the FA and choroidal hyperpermeability demonstrated by ICG suggests that intravitreal bevacizumab is efficacious and further investigation into both the possible role of VEGF in the pathogenesis of CSC and treatment of CSC with anti-VEGF agents is recommended [27] [28].

Comparative studies between anti-VEGF therapy and PDT treatment have been performed. Semeraro et al. report the effectiveness and safety of intravitreal bevacizumab injection (IVB) compared with low-fluence photodynamic therapy (L-PDT) in eyes with chronic central serous chorioretinopathy (CSC). This was a prospective comparative interventional study of 22 patients affected by chronic CSC. Patients were randomly assigned to Group 1 (12 patients treated with $1.25 \mathrm{mg}$ IVB) and Group 2 (10 patients treated with L-PDT). In Group 2, PDT with verteporfin was delivered at low fluence $\left(300 \mathrm{~mW} / \mathrm{cm}^{2}\right.$ for $\left.83 \mathrm{~s}, 25 \mathrm{~J} / \mathrm{cm}^{2}\right)$. Follow-up visits were scheduled at 1, 3, 6, and 9 months. The improvement in visual acuity was greater in Group 1 than in Group 2, although the difference was not statistically significant $(p=0.59$ ). Based on the results obtained after 9 months of follow-up, this study provides evidence that IVB may be a treatment option for chronic CSC [29].

Recently, Bae and colleagues compared half-fluence PDT to intravitreal ranibizumab (0.5 mg) in a randomized trial of 34 eyes with chronic CSC (defined as recurrent CSC or persistent fluid for 6 months). At month 12, 16 eyes (88.9\%) of the low-fluence PDT group maintained complete resolution of SRF without rescue treatment versus 2 eyes $(12.5 \%)$ in the ranibizumab group $(p<0.001)$. The improvement in BCVA from baseline was superior in the low-fluence PDT group to that in the ranibizumab group, but the differences were not statistically significant except at month $3(p=0.025)$. These findings in this small study suggest that half-fluence PDT may be superior to intravitreal ranibizumab as a treatment for chronic CSC and requires further investigation [30].

\section{Conclusions}

This study has several limitations: there is a tendency of CSC toward spontaneous remission, it is a small sample size and the lack of a control group.

Even though, a recent prospective randomized trial demonstrated superiority of reduced fluence PDT over anti-VEGF treatment (ranibizumab), regarding that CSC typically resolves on its own, leaving one to wonder whether therapy or time caused resolution. As well as, the lack of understanding about its etiology makes it unclear what the treatment target should be.

Although PDT caused the resolution of SRF in the majority of patients, improvements in VA of a similar magnitude were not observed [22]. On the other hand, PDT with verteporfin is an expensive therapy and it is not 
available in all countries.

Finally the evidence of multiples studies that demonstrated intravitreal bevacizumab effectiveness and safety in CSC; we concluded with these results that it is a valuable option therapy in patients with chronic and recurrent central serous choriorretinopathy.

\section{Brief Summary Statement}

The purpose of our study was to report the use of intravitreal bevacizumab as a therapeutic option in the treatment of chronic or recurrent central serous chorioretinopathy (CSC).

\section{References}

[1] Ober, M.D., Yannuzzi, L.A., Do, D.V., et al. (2005) Photodynamic Therapy for Focal Retinal Pigment Epithelial Leaks Secondary to Central Serous Chorioretinopathy. Ophthalmology, 112, 2088-2094. http://dx.doi.org/10.1016/j.ophtha.2005.06.026

[2] Yannuzzi, L.A., Slakter, J.S., Kaufman, S.R. and Gupta, K. (1992) Laser Treatment of Diffuse Retinal Pigment Epitheliopathy. European Journal of Ophthalmology, 2, 103-114.

[3] Cardillo Piccolino, F. and Borgia, L. (1994) Central Serous Chorioretinopathy and Indocyanine Green Angiography. Retina, 14, 231-242. http://dx.doi.org/10.1016/j.ophtha.2005.06.026

[4] Guyer, D.R., Yannuzzi, L.A., Slaktler, J.S., et al. (1994) Digital Indocyanine Green Videoangiography of Central Serous Chorioretinopathy. JAMA Ophthalmology, 112, 1057-1062. http://dx.doi.org/10.1001/archopht.1994.01090200063023

[5] Prunte, C. and Flammer, J. (1996) Choroidal Capillary and Venous Congestion in Central Serous Chorioratinopathy. American Journal of Ophthalmology, 121, 26-34.

[6] Cardillo Piccolino, F., Eandi, C.M., Ventre, L., et al. (2003) Photodynamic Therapy for Chronic Central Serous Chorioretinopathy. Retina, 23, 752-763. http://dx.doi.org/10.1097/00006982-200312000-00002

[7] Chan, W.M., Lam, D.S., Lai, T.Y., et al. (2003) Choroidal Vascular Remodeling in Central Serous Chorioretinopathy after Indocyanine Green Guided Photodynamic Therapy with Verteporfin: A Novel Treatment at the Primary Disease Level. British Journal of Ophthalmology, 87, 1453-1458. http://dx.doi.org/10.1136/bjo.87.12.1453

[8] Colucciello, M. (2006) Choroidal Neovascularization Complication Photodynamic Therapy for Central Serous Retinopathy. Retina, 26, 239-242. http://dx.doi.org/10.1097/00006982-200602000-00027

[9] Bujarborua, D. and Nagpal P. (2005) CSR: Idiopathic Central Serous Chorioretinopathy. Jaypee Brothers Medical Publishers, New Delhi.

[10] Leaver, P. and Willians, C. (1979) Argon Laser Photocoagulation in the Treatment of Central Serous Retinopathy. British Journal of Ophthalmology, 63, 674-677. http://dx.doi.org/10.1136/bjo.63.10.674

[11] Flicker, L., Vadifis, G., While, A. and Leaver, P. (1988) Long Term Follow up of a Prospective Trial of Argon Laser Photocoagulation in the Treatment of Central Serous Retinopathy. British Journal of Ophthalmology, 72, 829-834. http://dx.doi.org/10.1136/bjo.72.11.829

[12] Yannuzzi, L.A., Shakin, J.L., Fisher, Y.L. and Altomonte, M. (1984) Peripheral Detachments and Retinal Pigment Epithelial Atrophic Tracts Secondary to Central Serous Pigment Epitheliopathy. Ophthalmology, 91, 1553-1572. http://dx.doi.org/10.1016/S0161-6420(84)34117-3

[13] Jalk, A.E., Jabbour, N., Avila, M., Trempe, C.L. and Schepens, C.l. (1984) Retinal Pigment Epithelium Decompesation. II. Laser Treatment. Ophthalmology, 91, 1549-1553. http://dx.doi.org/10.1016/S0161-6420(84)34118-5

[14] Yannuzzi, L.A. (1989) Central Serous Chorioratinopathy. In: Yannuzzi, L.A., Ed., Laser Photocoagulation of the Macula, Lippincott, Philadelphia, 1-12.

[15] Battaglia Parodi, M., Da Pozzo, S. and Ravalico, G. (2003) Photodynamic Therapy in Chronic Central Seroschorioretinopathy. Retina, 23, 235-237. http://dx.doi.org/10.1097/00006982-200304000-00016

[16] Cardillo Piccolino, F., Eandi, C.M., Ventre, L., et al. (2003) Photodynamic Therapy for Chronic Central Seroschorioretinopathy. Retina, 23, 752-763. http://dx.doi.org/10.1097/00006982-200312000-00002

[17] Lai, T.Y., Chan, W.M. and Lam, D.S. (2004) Transient Reduction in Retinal Function Revealed by Multifocal Electroretinogram Following Photodynamic Therapy. American Journal of Ophthalmology, 137, 826-833. http://dx.doi.org/10.1016/j.ajo.2003.11.079

[18] Tzekov, R., Lin, T., Zhang, K.M., et al. (2006) Ocular Changes after Photodynamic Therapy. Investigative Ophthalmology Visual Science, 47, 377-385. http://dx.doi.org/10.1167/iovs.05-0838 
[19] Oner, A., Karakucuk, S., Mirza, E. and Erkilic, K. (2005) Electrooculography after Photodynamic Therapy. Documenta Ophthalmologica, 111, 83-86. http://dx.doi.org/10.1007/s10633-005-4410-4

[20] Oner, A., Karakucuk, S., Mirza, E. and Erkilic, K. (2005) The Changes of Pattern Electroretinography at the Early Stage of Photodynamic Therapy. Documenta Ophthalmologica, 111, 107-112. http://dx.doi.org/10.1007/s10633-005-4608-5

[21] Verma, L., Sinha, R., Venkatesh, P. and Tewari, H.K. (2004) Comparative Evaluation of Diode Laser versus Argon laser Photocoagulation in Patients with Central Serous Retinopathy: A Pilot, Randomized Controlled Trial. BMC Ophthalmology, 4, 15. http://dx.doi.org/10.1186/1471-2415-4-15

[22] Lim, J.I., Glassman, A.R., Aiello, L.P., et al. (2014) Collaborative Retrospective Macula Society Study of Photodynamic Therapy for Chronic Central Serous Chorioretinopathy. Ophthalmology, 121, 1073-1078. http://dx.doi.org/10.1016/j.ophtha.2013.11.040

[23] Niegel, M.F., Schrage, N.F., Christmann, S. and Degenring, R.F. (2008) Intravitreal Bevacizumab for Chronic Central Serous Chorioretinopathy. Ophthalmologe, 105, 943-945. http://dx.doi.org/10.1016/j.ophtha.2013.11.040

[24] Artunay, O., Yuzbasioglu, E., Rasier, R., et al. (2010) Intravitreal Bevacizumab in Treatment of Idiopathic Persistent Central Serous Chorioretinopathy: A Prospective, Controlled Clinical Study. Current Eye Research, 35, 91-98. http://dx.doi.org/10.3109/02713680903428306

[25] Lijima, H., Lida, T., Murayama, K., et al. (1999) Plasminogen Activator Inhibitor 1 in Central Seroschorioretinopathy. American Journal of Ophthalmology, 127, 477-478. http://dx.doi.org/10.1016/S0002-9394(98)00378-X

[26] Shahar, J., Avery, R.L., Heilweil, G., et al. (2006) Electrophysiologic and Retinal Penetration Studies Following Intravitreal Injection of Bevacizumab (Avastin). Retina, 26, 262-269. http://dx.doi.org/10.1097/00006982-200603000-00002

[27] Lim, J.W., Kim, M.U. and Shin, M.C. (2010) Aqueous Humor and Plasma Levels of Vascular Endothelial Growth Factor and Interleukin-8 in Patients with Central Serous Chorioretinopathy. Retina, 30, 1465-1471. http://dx.doi.org/10.1097/IAE.0b013e3181d8e7fe

[28] Shin, M.C. and Lim, J.W. (2011) Concentration of Cytokines in the Aqueous Humor of Patients with Central Serous Chorioretinopathy. Retina, 31, 1937-1943. http://dx.doi.org/10.1097/IAE.0b013e3181d8e7fe

[29] Semeraro, F., Romano, M.R., Danzi, P., Morescalchi, F. and Costagliola, C. (2012) Intravitreal Bevacizumab versus Low-Fluence Photodynamic Therapy for Treatment of Chronic Central Serous Chorioretinopathy. Japanese Journal of Ophthalmology, 56, 608-612. http://dx.doi.org/10.1007/s10384-012-0162-3

[30] Bae, S.H., Heo, J., Kim, C., Kim, T.W., Shin, J.Y., Lee, J.Y., Song, S.J., Park, T.K., Moon, S.W. and Chung, H. (2014) Low-Fluence Photodynamic Therapy versus Ranibizumab for Chronic Central Serous Chorioretinopathy. Ophthalmology, 121, 558-565. http://dx.doi.org/10.1016/j.ophtha.2013.09.024 
Scientific Research Publishing (SCIRP) is one of the largest Open Access journal publishers. It is currently publishing more than 200 open access, online, peer-reviewed journals covering a wide range of academic disciplines. SCIRP serves the worldwide academic communities and contributes to the progress and application of science with its publication.

Other selected journals from SCIRP are listed as below. Submit your manuscript to us via either submit@scirp.org or Online Submission Portal.
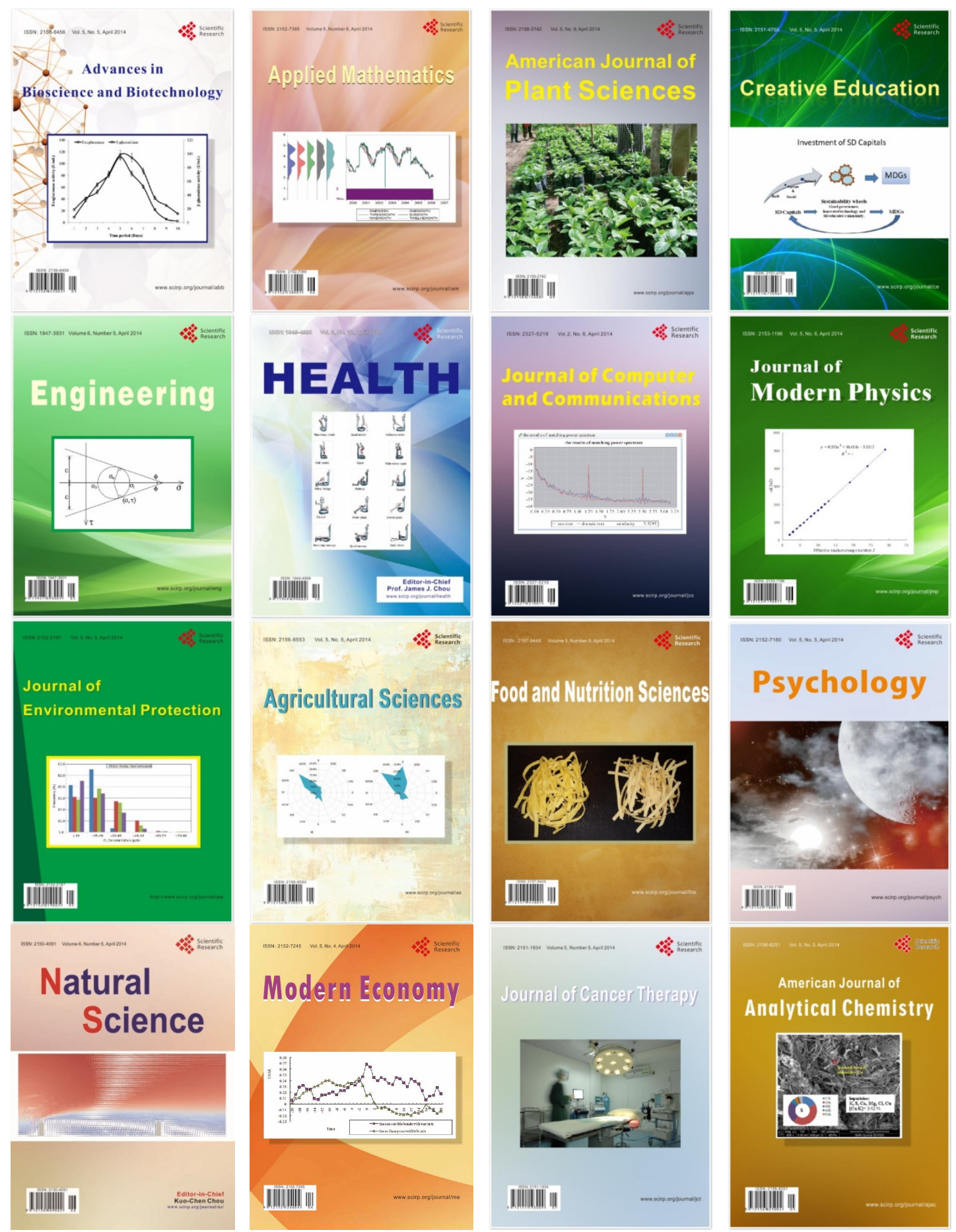